\title{
ADHD and DIRAS Single Nucleotide Polymorphism as an Indicator of Prolonged Concussion Recovery
}

TAZIAH KENNEY ${ }^{1}$ JANE MCDEVIT'T ${ }^{2}$

${ }^{1}$ College of Life Sciences, Jefferson University ${ }^{2}$ Department of Health and Rehabilitation Sciences, College of Public Health, Temple University

Correspondence: Kenney.Taziah@gmail.com (Taziah Kenney)

\begin{abstract}
Context: The purpose of this study was to determine the association between the presence of a single nucleotide polymorphism (SNP; rs1412005) within the promoter region of DIRAS2 (i.e., a gene associated with $A D H D)$ and the association with prolonged recovery following a sport-related concussion. Methods: We implemented a case-control study, where cases and controls were selected from a cohort of 117 deidentified concussed athletes (70\% male, 20+5.22 y/o; 21 different sports). Eleven samples from this patient cohort conveyed a self-reported diagnosed $A D H D$ and were age and sex-matched to 22 participants with no self-reported ADHD diagnoses. The DNA from saliva samples was extracted, estimated, and genotyped using real-time polymerase chain reaction. Results: The average recovery time for the case group was 21.50 days $(S D=13.96)$, while the average recovery time for the control group was 15.66 days $(S D=8.50)$. We found that individuals with $A D H D$ were 5 times more likely to have prolonged recovery than their non-ADHD counterparts $(C I=.95)$. Also, the authors found that only $13.6 \%$ of the individuals without an ADHD diagnosis recovered in $>30$ days $(p=0.044)$. Additionally, the authors found that $72.7 \%$ of the carriers of the $T$ allele (i.e., minor allele) recovered in greater than 30 days ( $p=0.213)$. Conclusion: Researchers concluded that individuals with ADHD had a bigher risk of prolonged concussion recovery lasting greater than 30 days. Also, carrying the rare allele was associated with prolonged recovery, which suggests this $S N P$ could be a potential genetic marker for both prolonged concussion recovery and the presence of $A D H D$.
\end{abstract}

Keywords: ADHD, concussion, genetic variation, concussion recovery

\section{Concussion Recovery}

Ten percent of the population has an attention deficit hyperactivity disorder (ADHD) diagnosis, ${ }^{1}$ which mirrors the presence of this condition among athletes. ${ }^{2}$ Attention Deficit Hyperactivity Disorder (ADHD) is characterized by symptoms of inattention, hyperactivity, and impulsivity. ${ }^{3}$ Researchers previously attributed ADHD to functional brain abnormalities of the pre-frontal cortex and basal ganglia. ${ }^{4}$ More recently, a wide-ranging metaanalysis of functional magnetic resonance imaging (fMRI) studies, concluded that there were many variations in the functional networks of the brain that may contribute to ADHD symptoms. ${ }^{5}$ Although there is still speculation about the areas of the brain primarily responsible for $\mathrm{ADHD}$, stimulant treatments (e.g., methylphenidates) have been most 
successful in treating its symptoms. Methylphenidate medication causes an alteration in the dopaminergic processes in the brain, leading to a symptom resolution in individuals with ADHD. ${ }^{6}$ Dopaminergic processes involve the release or inhibition of the neurotransmitter dopamine. Dopamine is present in the forebrain and basal ganglia, where it contributes to both motor and limbic processes (e.g., thinking processes). ${ }^{6}$ Dopamine is also abundant in the frontal lobes, which primarily regulate both cognitive (e.g., working memory) and information processes. $^{6}$ Individuals with ADHD have imbalances within the dopaminergic pathways in the brain which may contribute to dysfunction with cognitive and memory processes. ${ }^{6}$

Concussion, like ADHD, presents with symptoms of both cognitive and memory complications, which may be due to ionic imbalances and an increase in neurotransmitters released in the brain (e.g., dopamine and glutamate). ${ }^{7}$ Concussion is a growing public health concern due to the potential for deleterious and long-term cognitive effects. ${ }^{8}$ Approximately 10 million patients visit the emergency room annually due to a concussion. ${ }^{8}$ Of those, 3.8 million obtain a concussion from sport-related activities. ${ }^{9}$ Typically, concussion symptoms resolve within 7-10 days, and high school and college age patients may fully return to play within 11-20 days. ${ }^{11,12}$ However, some individuals may experience prolonged recover post-concussion. While some researchers consider concussion symptoms that last 30 days or more to be indicative of prolonged recovery, ${ }^{13,}{ }^{14}$ though this is not well defined. The presence of a learning disability (e.g., ADHD) and genetics (i.e., single nucleotide polymorphisms; SNPs) are risk factors for prolonged recovery time post-concussion. ${ }^{10}$ Other risk factors for prolonged recovery from concussion include learning disabilities (ADHD, previous concussions, sex, and age. ${ }^{10}$ Prolonged concussion recovery time and increased risk of a concussion are common for patients with ADHD. ${ }^{10,16-18}$ Assessing patients for intrinsic risk factors, such as ADHD, is important when considering concussion recovery. Another intrinsic risk factor that has not been fully parsed out is a patient's genome. Genotype in certain genetic markers may play a role in both concussion recovery and hereditary of learning disabilities such as ADHD. ${ }^{10,19}$

A single nucleotide polymorphism (SNP; i.e., a one nucleotide change within the genome) is denoted by rs1412005 within the promoter region of the Distant Subgroup of The Ras Family Member 2 (DIRAS2) gene is associated with ADHD diagnosis. ${ }^{19}, 20$ The DIRAS2 regulates over 1,600 other genes, many of which have primary roles in neuronal proliferation and alteration. ${ }^{21} \mathrm{~A}$ knockout study (i.e., a study where a gene is isolated and to determine the impact when a gene is no longer present) of DIRAS2 in murine hippocampal primary cells concluded that overexpression of DIRAS2 was a common occurrence in individuals with ADHD due to the presence of the risk allele (i.e., T). ${ }^{21,22}$ The investigation of SNPs linked to neuronal recovery has been a growing area of research in prolonged concussion recovery. Recently, a repeat polymorphism within the glutamate receptor ionotropic 2A (GRIN2A) gene was detected, and researchers found that carriers with 25 or more GT repeats were associated with a six-fold increased risk of concussion recovery lasting 60 or more days. ${ }^{23}$ Additionally, the Apolipoprotein (APOE) E4 allele has been found to be associated with increased symptom reporting and higher recovery time following a sport-related concussion. ${ }^{24}$

There has been no research to determine an association between the alleles (i.e., $G, T$ ) within the DIRAS2, ADHD, and recovery time for sport-related concussion in the athletic population. If the rare allele (i.e., Thymine; T) polymorphism is found to be associated with an ADHD diagnosis, this may help medical professionals predict timing for recovery and safe return to play for athletes with ADHD. Therefore, the purpose of this study was to determine the association between the presence of the risk allele (i.e., T) in DIRAS2 and prolonged recovery (> 30 days) from sportrelated concussion. 


\section{Method}

\section{Participants}

This case-control study was designed to assess the presence of prolonged concussion recovery and the rare allele (i.e., T) within a cohort of individuals with ADHD and their non-ADHD counterparts. Case participants were defined as participants with self-reported ADHD. The control participants were defined as participants with no self-reported ADHD. Participants included 11 case individuals, matched by age and sex to 22 control individuals without ADHD ( 2 matched controls per case). Participants were recruited from 2 different sites (i.e. Temple University and Temple Hospital). All participants in this study had suffered a sports-related concussion. The average age of participants was 18 years $(\mathrm{SD}=3)$ there were 30 males and 3 females and $70 \%$ of the sample population was Caucasian. Table 1 includes complete demographic information, as well as the sports played by participants. The average time to recovery from a sport-related concussion for the entire cohort of individuals was 17.27 days $(\mathrm{SD}=11.23)$ The average concussion recovery time for the case participants was 21.5 days $(\mathrm{SD}=13.96)$ while the control participants recovered within an average of 15.66 days $(\mathrm{SD}=8.50)$. Further demographic information can be found in Table 1 .

\section{Measures}

Participants completed a standardized initial concussion evaluation, where the following parameters were assessed: injury characteristics including the date and time of injury, mechanism of injury (e.g., direct impact, rapid acceleration, and deceleration), acute signs and symptoms (e.g., loss of consciousness, dizziness), patient history including selfreported prior concussion history, migraine, ADHD disclosure, and psychiatric history (e.g., depression). The initial evaluation was followed by an objective screening, which included vestibular ocular assessments, the Balance Error Scoring System (BESS) test, and an online

\begin{tabular}{|c|c|c|c|}
\hline & Total & Case & Control \\
\hline \multirow[t]{3}{*}{$\begin{array}{l}\text { Factor } \\
\text { Sex }\end{array}$} & 33 & \multicolumn{2}{|c|}{ n (\%) } \\
\hline & Male & $10(91.00)$ & 10 (91.00) \\
\hline & Female & $1(9.00)$ & $1(9.00)$ \\
\hline \multicolumn{4}{|l|}{ Race } \\
\hline & Caucasian & 8 (81.81) & $13(59.09)$ \\
\hline & African American & 0 & $5(22.72)$ \\
\hline & Hispanic/Latinx & 0 & $1(4.54)$ \\
\hline & Native American & $1(9.09)$ & 0 \\
\hline & Not Given & $1(9.09)$ & $3(13.63)$ \\
\hline \multicolumn{4}{|l|}{ Sport } \\
\hline & Football & $5(45.45)$ & $8(36.36)$ \\
\hline & Rugby & $1(9.09)$ & $3(13.63)$ \\
\hline & Baseball & $1(9.09)$ & $1(4.54)$ \\
\hline & Basketball & $1(9.09)$ & $1(4.54)$ \\
\hline & Ice Hockey & $1(9.09)$ & $1(4.54)$ \\
\hline & Soccer & $1(9.09)$ & $5(22.76)$ \\
\hline & Fencing & 0 & $1(4.54)$ \\
\hline & \multicolumn{3}{|c|}{ Mean \pm SD } \\
\hline Age (yr) & $18 \pm 3$ & $18 \pm 3$ & $18 \pm 3$ \\
\hline MR (d) & $19 \pm 12$ & $22 \pm 14$ & $16 \pm 9$ \\
\hline PDC & $2 \pm 1$ & $1 \pm 2$ & $3 \pm 2$ \\
\hline
\end{tabular}

Note. Mean recovery (MR) is measured in days (d) and dictates mean recovery from sport-related concussion and PDC dictates previous diagnosed concussions.

Immediate Post-Concussion Assessment and Cognitive Testing (ImPACT). Previously recorded baseline scores were used to determine an athlete's readiness to return to play. Using these data, for each participant we assessed number of days to full recovery and status of ADHD diagnosis.

\section{DNA Extraction and Genotyping}

This SNP rs1412005 was chosen based on its primary expression within the brain, association with ADHD, and the minor allele frequency of greater than or equal to 0.40 . The SNP rs1412005 is located in the promoter 
region of DIRAS2, which is located on chromosome 9 in the promoter region. The promotor region of DIRAS2 has possible risk of the target SNP having some phenotypic response compared to choosing a SNP in the intron region of a gene. ${ }^{25}$ The promoter region of this gene dictates the amount of small Ras GTPase protein that is produced. Small Ras GTPase proteins play an important role in cell development, discrimination, and survival. ${ }^{25}$

Salivary samples for genotyping and DNA isolation were collected using Oragene DNA Self Collection Kits (DNA Genotek, Ottawa, ON, Canada). DNA was extracted according to the manufacturer's instructions. Purified DNA was quantified using a Quant-it PicoGreen DNA assay kit (Invitrogen, Carlsbad, CA). The DNA region surrounding position in the promoter of DIRAS2 was amplified (taqman assay kit number C_33069317_10). The cycling conditions were incubation for $30 \mathrm{~min}$ at $48^{\circ} \mathrm{C}, 10 \mathrm{~min}$ at $95^{\circ} \mathrm{C}$, followed by 40 cycles of $15 \mathrm{sec}$ at $95^{\circ} \mathrm{C}$ and 1 min at $60^{\circ} \mathrm{C}$. Real Time-Polymerase Chain Reaction was terminated after 35 cycles and all genotyping measures were read at 30 cycles to ensure the most accurate allelic discrimination. To validate the analytical method for SNP

\section{Results}

\section{Concussion and ADHD}

An odds ratio test revealed that individuals in the study with self-reported ADHD were five times more likely to have prolonged recovery than their non-ADHD counterparts (CI= 95\%) A chi-square test revealed a significant difference between presence of ADHD and concussion recovery $(p=0.044)$. Forty-five percent of individuals with ADHD had prolonged recovery from concussion, while $54 \%$ of the individuals with ADHD had normal concussion recovery.

Eighty-six percent of the individuals with no presence of ADHD had normal concussion recovery time (i.e., less than 30 days) and only $13 \%$ of those individuals had prolonged concussion recovery. This suggests that patients with ADHD have a significantly longer recovery genotyping, a pedigree analysis of both the $\mathrm{G} / \mathrm{T}$ alleles in a three-generation family was performed. The pedigree chart analysis confirmed that the protocol in this work was accurate in detecting both the homo- and heterozygous allelic variants and that the corresponding alleles were inherited according to Mendel's laws. Additionally, gel electrophoresis was used to quantify and check the purity of the reaction process using 1.4\% agarose. After electrophoresis at $100 \mathrm{mV}$ for 1 $\mathrm{hr}$, the gels were inspected under UV light, and the images were recorded.

Data were analyzed using descriptive and inferential statistics. A one-way Analysis of variance (ANOVA) was used to identify the association between number of days to full concussion recovery, ADHD diagnosis, and allele frequency. An odds ratio was utilized to predict the relative odds between prolonged recovery for individuals with ADHD and their non-ADHD counterparts. Three 2 (allele) x 2 (group) chi-square tests were used to assess the association between the allele frequency of DIRAS2 SNP, ADHD diagnosis status, and prolonged recovery. SPSS version 26.0 (SPSS IBM Inc., Armonk, NY) was used for all analyses, and the alpha level was set to $p \leq 0.05$.

time post-concussion, compared to patients without ADHD.

\section{ADHD and DIRAS2 Genotype}

There was no significant difference between the presence of ADHD and the rare allele genotype (i.e., GT; $p=0.213$ ), see results in Table 2.

\begin{tabular}{|c|c|c|c|}
\hline \multicolumn{4}{|c|}{$\begin{array}{l}\text { Table } 2 . \\
\text { ADHD Diagnosis, Genotype, and Prolonged Recovery } \\
\text { Relationship }\end{array}$} \\
\hline Factor & Outcome & Case & Control \\
\hline Total Sample & & 11 & 22 \\
\hline Allele & $\% G$ & 27.30 & 50.00 \\
\hline & $\% \mathrm{~T}$ & 72.70 & 50.00 \\
\hline Recovery & $\%$ Normal & 54.40 & 86.40 \\
\hline & $\%$ Prolonged & 45.50 & $13.60^{*}$ \\
\hline
\end{tabular}


and Figure 1. Seventy-two percent of individuals with the GT genotype had an ADHD diagnosis.

\section{Figure 1.}

Relationship Between Presence of ADHD and Sport-Related Concussion Recovery Time.

ADHD and Recovery Time

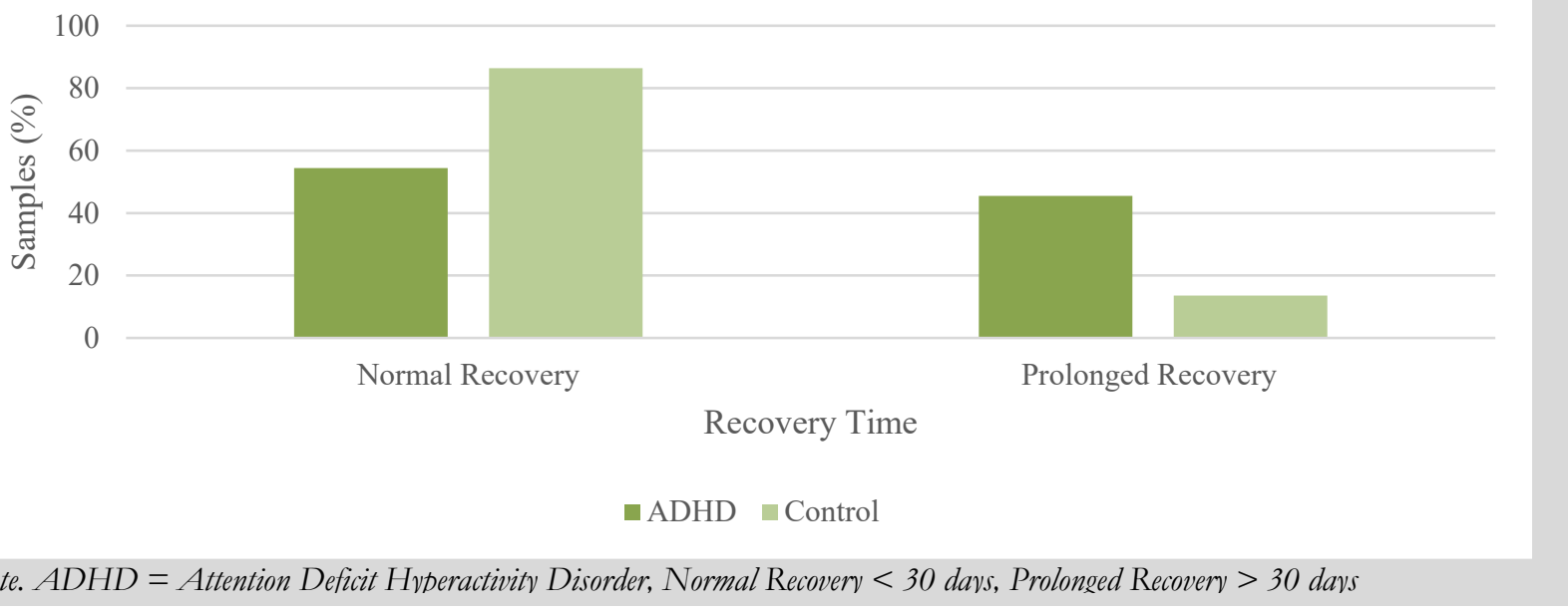

\section{Concussion Recovery and DIRAS2 Genotype}

A chi-square test between genotype and concussion recovery revealed no significant difference for genotype and prolonged concussion recovery $(p=.0 .252)$. Additional information about the relationship between concussion recovery and genotype can be seen in Table 2 .

\section{ADHD, Genotype, and Concussion Recovery}

There was no significant association between days to full concussion recovery, the presence of ADHD, and genotype $(p=0.143)$. The relationship between self-reported ADHD status, genotype, and concussion recovery can be seen in Figure 2.

\section{Figure 2.}

Relationship Between Genotype and Recovery Time

\section{Genotype and Recovery Time}

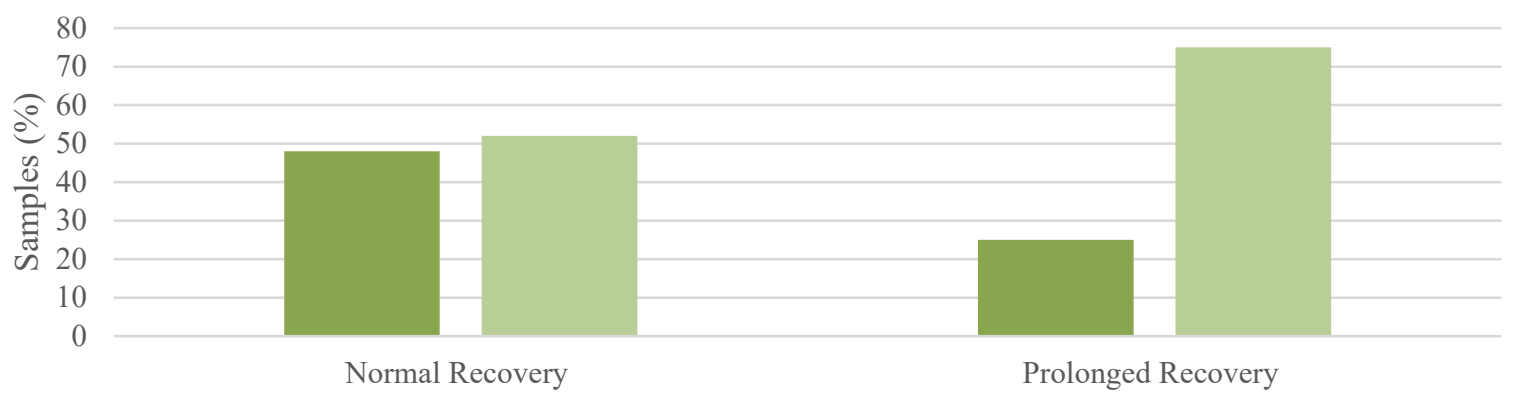

Recovery Time

$\varpi \mathrm{G} \pm \mathrm{T}$

Note. $G G=$ Common genotype, $G T=$ Rare genotype 


\section{Discussion}

A variety of risk factors may contribute to the probability of prolonged recovery following sport-related concussion. This study sought to investigate the presence of the rare allele (i.e., $\mathrm{T}$ ) in the promoter SNP within DIRAS2, ADHD, and their association with concussion recovery lasting 30 or more days. Individuals with ADHD were found to be five times more likely to suffer from a prolonged concussion recovery $(\mathrm{CI}=95 \%)$ compared to their non-ADHD counterparts. The American Society for Sports Medicine position statement on concussion in sport described ADHD as a risk factor for abnormal concussion outcomes due to its association with cognitive dysfunction. ${ }^{9}$ A study comparing recovery time for individuals with ADHD and their non-ADHD counterparts found a three-day difference between the patients, solely based on their ADHD status. ${ }^{17}$ Miller and colleagues also found ADHD to be a predicator for prolonged concussion recovery of greater than 28 days. ${ }^{26}$ This may be due to an exacerbation of athletes' ADHD symptoms because of the common symptomology between concussions and ADHD. ${ }^{7}$

Attention Deficit Hyperactivity Disorder status was not significantly predictive of a higher likelihood of the presence of the rare allele, $\mathrm{T}$, within this cohort of 33 athletes $(72.7 \%$ in cases vs. $50 \%$ in controls). The DIRAS2 rare allele, T has been previously associated with an ADHD diagnosis. ${ }^{20}$ The minor risk allele led to the increased expression of DIRAS2. ${ }^{22}$ DIRAS2 RNA expression was found to be heavily expressed in the hippocampus, cerebral cortex, and the cerebellum, all regions of the brain responsible for cognitive and memory function. ${ }^{22}$ Use of the western blot technique found the highest expression levels of DIRAS2 in the frontal cortex, hippocampus, amygdala, and striatum of the brain ${ }^{22}$ which are areas of the brain involved in working memory,

\section{Limitations and Future Research}

Although there was an association between prolonged concussion recovery and the presence of ADHD, the demographic information error detection, executive function. ${ }^{19}$ Specifically, the neurotransmitters dopamine and glutamine within the striatum were found to have an association with altered cognitive function. ${ }^{7}$

Seventy-five percent of individuals that disclosed a diagnosis of ADHD and were found to be carrying the $\mathrm{T}$ allele had prolonged recovery, while only $25 \%$ of the controls experienced this outcome. Additionally, researchers found that $100 \%$ of the individuals with the $\mathrm{G}$ allele recovered in 30 days or fewer (Figure 2). The rare allele $\mathrm{T}$ has been found to be associated with the overexpression of DIRAS2, which plays a role in the regulation of 1,612 genes. ${ }^{21}$ Of the genes regulated by DIRAS2, many were found to play a role in processes involving glutamate. ${ }^{22}$ Glutamate is a key neurotransmitter in the neurometabolic cascade that occurs following a concussion. ${ }^{27}$ The Neurometabolic Cascade theory following neuronal strain suggests that the release of glutamate, as an excitatory neurotransmitter, promotes accumulation and influx of intracellular sodium and calcium. This leads to cell and mitochondrial damage within the neurons. ${ }^{27}$ Due to the increased need for homeostasis propagated by the mitochondria, there is a subsequent energy crisis that occurs. The energy crisis has been shown to propagate poorer cognitive function in animal studies.27 Glutamate has also been found to be associated with alterations with the neurotransmitter dopamine. A study found that the administration of glutamate into the ventral striatum reduces the uptake of dopamine? Dopamine has been an important neurotransmitter involved in the symptomology of ADHD. Dopamine plays an important role in motor control, motivation, cognitive functions, and other functions of the body. ${ }^{28}$ Due to the relationship between glutamate and dopamine, concussion impact may be more debilitating for individuals with an ADHD diagnosis, which may lead to prolonged concussion recovery.

may have been affected by recall bias. For example, the presence of ADHD was self-reported at the beginning of the study. Some participants may have 
chosen not to self-report their ADHD diagnosis status, or their diagnosis could have changed throughout the duration of the study. Further research with a larger and more diverse population is necessary to determine the overall significance of this SNP. To determine a widespread genome significance, it would be necessary to conduct a genome-wide analysis study examining the

\section{Clinical Implications}

Individuals with ADHD have a higher likelihood of prolonged concussion recovery, which may be associated with the presence of the rare allele (i.e., T) within DIRAS2. The DIRAS2 protein is highly expressed in brain regions that play a role in genes that regulate dopamine and relationship between $D I R A S 2$ and genes that it affects as a proliferation switch. Lastly, the control group reported more previous concussions, a contributing risk factor for prolonged concussion recovery, than our case group, which may have affected our findings. ${ }^{17,18}$

\section{About the Authors}

Taziah Kenney is a visiting assistant professor within the College of Life Sciences at Thomas Jefferson University. She earned her M.S in Kinesiology at Temple University. Currently, she is pursuing a Doctor of Education with a line of research focused on student success and retention in special populations.

Jane McDevitt is an assistant of instruction in the Department of Health and Rehabilitation Sciences at Temple University. She earned her PhD in Kinesiology at Temple University. Currently, she is the director of the Sport Concussion Lab, where her primary line of research is in the risks of prolonged recovery following a concussion.

\section{Conflicts of Interest}

The authors declare no conflicts of interest.

\section{Statement of Contributions}

T.K. and J.M. contributed to the design and implementation of the research, the analysis of the results, and the writing and editing of the manuscript.

\section{References}

1. Alosco, M, Fedor, A, and Gunstad, J, Attention Deficit Hyperactivity Disorder as a Risk Factor for Concussions in NCAA division-I athletes. Brain Injury. 2014; 28(4): 2-4

2. Matuszak, J. Attention Deficit/Hyperactivity Disorder (ADHD). Mental Health in the Athlete. 2020; pp 66-84

3. Young, JL, Goodman, DW. Adult Attention-Deficit/Hyperactivity Disorder Diagnosis, Management and Treatment in DSM-5 Era. Journal of Primary Care Companion CNS Disorders 2016; 18(6)

4. Friedman, L, Rapoport, J. Brain development in ADHD. Current Opinion in Neurobiology 2015; 30:106-111. doi. 10.1016/j.conb.2014.11.2007 
5. Cortese, S, Kelly, C, Chabernaud, C, Proal, E, and Martino, A. Toward Systems Neuroscience of ADHD: A meta-analysis of 55 fMRI studies. The American Journal of Psychiatry. 2012; 169(10): 10381055

6. Alexander, L, Farrelly, N. Attending to adult ADHD: A review of the neurobiology behind adult ADHD. Irish Journal of Psychological Medicine. 2017; pp. 237-244

7. Gleich, T, Desnerno, L, Lorenz, R, Boehme, R, Pankow, A, Buchert, R, Kühn, S, Heinz, A, Schlagenhauf, F, Gallinat, J. Prefronal and striatal glutamate differently relate to striatal dopamine: Potential regulatory mechanisms of striatal presynaptic dopamine function. Journal of Neuroscience. 2015; 35(26): 9615-9621

8. Langlois, J, Rutland-Brown, W, Wald, M. The epidemiology and impact of traumatic brain injury: A brief overview. Journal of Head Trauma Rehabilitation. 2006; 21(5): 375-378

9. Harmon, K, Drezner, J, Gammons, M, Guskiewics, K, Halstead, M, Herring, S, Kutcher, J, Pana, A, Roberts, W. American Medical Society for Sports Medicine position statement: concussion in sport. British Journal of Sports Medicine. 2013; 47(1)

10. Iverson, GL, Gardner, AJ, Terry, DP, Ponsford, J, Sills, A, Broshek, D, Solomon, GS. Predictors of clinical recovery from concussion: A systematic review. British Journal of Sports Medicine. 2017; 51(12): 941-948.

11. Covassin, T, Bretzin, A, Beidler, E, Wallace, J. Time-to-Event analyses: Return to unrestricted participation following sport-related concussion in a cohort of high school athletes. Journal of athletic training. 2020; doi: https://doi.org/10.4085/150-20

12. Tamura, K, Furutani, T, Oshiro, R, Oba, Y, Ling, A, Murata, N. Concussion Recovery Timeline of High School Athletes Using A Stepwise Return-to-Play Protocol: Age and Sex Effects. Journal of Athletic Training. 2021; 55(1), 6-10. https://doi.org/10.4085/1062-6050-452-18

13. Giza, C, Prins, M, Hovda, D. It's not all fun and games: Sports, concussions, and neuroscience. Neuron. 2017; 94(6):1051-1055

14. Leddy, J, Baker, J, Nadir Haider, M, Hinds, A, Willer, B. A physiological approach to prolonged recovery from sport-related concussion. Journal of Athletic Training. 2017; 52(3): 299-308

15. Master C, Master S, Wiebe D, Storey E, Lockyer J, Podolak O, Grady M. Vision and Vestibular System Dysfunction Predicts Prolonged Concussion Recovery in Children. Clinical Journal of Sport Medicine 2018; 28(2): 139-145.

16. Bonfield, C, Lam, S, Lin, Y, Greene, S. The impact of attention deficit hyperactivity disorder on recovery from mild traumatic brain injury. Journal of Neurosurgery Pediatrics. 2013; 12(2): 97-102

17. Mautner, K, Sussman, WI, Axtman, M, Al-Farsi, Y, Al-Adawi, S. Relationship of Attention Deficit Hyperactivity Disorder and Post-concussion Recovery in Youth Athletes. Clinical Journal of Sports Medicine. 2015; 25(4): 355-60

18. Wolfe, E, Madden, K. Evidence-based considerations for athletic trainers caring for patients with attention deficit/hyperactivity disorder. Journal of Athletic Training. 2016; 51(10): 813-820

19. Reif, A, Nguygen, T, Weibflog, L, Jacob, C, Romanos, M, Renner, T, Buttenschon, H, et al. DIRAS2 is associated with adult ADHD, related traits, and co-morbid disorders. Neuropsychopharmacology. 2011; 36(11): 2318-2327

20. Grünewald, L, Landaas, E, Geissler, J, Weber, H, Quast, C, Röh, S, Schartner,C, Lesch, K, Romanos, M, Kittel-Schneider, S, Binder, E, Reif, A. Functional impact of an ADHD-associated DIRAS2 promoter polymorphism. Neuropsychopharmacology. 2016; 41: 3025-3031

21. Grünewald, L, Chiocchetti, A, Weber, H, Scholz, C, Schartner, C, Freudenberg, F, Reif, A. Knockdown of the ADHD candidate gene Diras2 in murine hippocampal primary cells. Journal of Attention Disorders 2019; [Epub]

22. Grünewald, L, Becker, N, Camphausen, A, O'Leary, A, Klaus-Peter, L, Freudenberg, F, Reif, A. Expression of the ADHD candidate gene DIRAS2 in the brain. Journal of Neural Transmission. 2018;125(6): 913-923. 
23. McDevitt, J, Tierney, R, Phillips, J, Gaughan, J, Torg, J, Krynetskiy, E. Association between GRIN2A promoter polymorphism and recovery from concussion. Brain Injury. 2015; 29: 13-14

24. Merritt, V, Rabinowitz, A, Arnett, P. The influence of the Apolipoprotein E (APOE) gene on subacute post-concussion neurocognitive performance in college athletes. Clinical Neuropsychology. 2017; 33(1): 36-46

25. Schöpel, M, Potheraveedu, V, Al-Harthy, T, Abdel-Jalil, R, Heumann, R, Stoll, R. The small GTPases Ras and Rheb studied by multidimensional NMR spectroscopy: Structure and function. Biological Chemistry. 2017; 398(5-6)

26. Miller, J, Gill, C, Kuhn, E, Rocque, B, Menedez, J, O’Neil, J, Agee, B, Brown, S, Crowther, M, Davis, R, Ferguson, D, Johnston, J. Predictors of delayed recovery following pediatric sports-related concussion: a case-control study. Journal of neurosurgery pediatrics. 2016; 17(4): 491-6

27. Romeu-Mejia, R, Giza, C, Goldman, J. Concussion pathophysiology and injury biomechanics. Current reviews in musculoskeletal medicine. 2019; 12(2): 105-116

28. Klein, M, Battagello, D, Cardoso, A, Hauser, D, Bittencourt, J, Correa, R. Dopamine: Functions, signaling, and associations with neurological diseases. Cell Molecule Neurobiology. 2019; 39(1):31-59 